\title{
RESOLUÇÃO NUMÉRICA DA EQUAÇÃO DIFERENCIAL DE RESFRIAMENTO DE NEWTON PELOS MÉTODOS DE EULER E RUNGE- KUTTA
}

\author{
A. Pescador ${ }^{1}$; Z.R.Q. Oliveira ${ }^{2}$ \\ 1 Universidade Federal de Santa Catarina, Florianópolis - SC - 88040-900, Brasil \\ 2 Instituto Federal Catarinense, Sombrio, SC - 88960-000 - Brasil \\ * andresa.pescador@gmail.com
}

\section{RESUMO}

O presente artigo apresenta as equações diferenciais de primeira ordem, as quais constituem um ramo muito importante da matemática, pois têm uma grande aplicabilidade, tanto na matemática, como na física, biologia e também na economia. O objetivo deste estudo foi analisar a resolução de uma equação diferencial de primeira ordem, em especial a equação que define a lei de resfriamento de Newton. Verificar seu comportamento utilizando algumas aplicações, que podem ser utilizadas em sala de aula como instrumento de auxílio ao professor
\end{abstract}

na abordagem destes conteúdos trazendo respostas aos questionamentos dos estudantes e motivando-os na construção de seu conhecimento. Para a resolução de uma das aplicações apresentadas buscou-se como complemento sua resolução através de dois métodos numéricos, método de Euler e método de Runge-Kutta. E por fim, fez-se uma comparação da aproximação da solução dada pela resolução numérica com a resolução analítica cuja solução é exata.

PALAVRAS-CHAVE: equações diferencias, aplicações, métodos numéricos, solução analítica, solução numérica.

\section{NUMERICAL SOLUTION OF NEWTON'S COOLING DIFFERENTIAL EQUATION BY THE METHODS OF EULER AND RUNGE -KUTTA}

\begin{abstract}
This article presents the first-order differential equations, which are a very important branch of mathematics as they have a wide applicability, in mathematics, as in physics, biology and economy. The objective of this study was to analyze the resolution of the equation that defines the cooling Newton's law. Verify its behavior using some applications that can be used in the classroom as an auxiliary instrument to the teacher in addressing
\end{abstract}

these contents bringing answers to the questions of the students and motivating them to build their knowledge. It attempted to its resolution through two numerical methods, Euler method and Runge Kutta method. Finally, there was a comparison of the approach of the solution given by the numerical solution with the analytical resolution whose solution is accurate.

KEYWORDS: differential equations , applications, numerical methods, analytical solution, numerical solution . 


\section{INTRODUÇÃO}

Faz parte do cotidiano dos professores, em especial professores de matemática, ouvir de seus estudantes questionamentos que se referem ao conteúdo ministrado com relação a sua aplicabilidade. Os estudantes querem saber para que serve e onde podem aplicar tais conteúdos, mas nem sempre ficam satisfatoriamente esclarecidos. Mesmo que a matemática não deva ser trabalhada para satisfazer tais ensejos, aplicações práticas enobrecem, estimulam e motivam os estudantes para o seu aprendizado.

Acreditando-se que o ensino de alguns conteúdos de matemática possa ser estreado com uma aplicação do tema para motivação ou ainda concluído com uma aplicação para o fechamento, este trabalho traz aplicações relacionadas ao conteúdo de equações diferenciais onde se fez a escolha do tema Lei de Resfriamento de Newton.

A situação proposta é desenvolvida apresentando-se alguns exemplos práticos sobre a Lei de Resfriamento de Newton. Em seguida resolve-se a equação diferencial de forma analítica e por fim resolve-se a mesma por dois métodos numéricos clássicos e comparam-se os três resultados encontrados.

Desta forma, na tentativa de responder aos questionamentos apresentados pelos estudantes, faz-se a inserção de situações problemas, ainda com intuito de trazer o interesse dos estudantes ao conteúdo. Neste, escolhe-se o tema de equações diferenciais, na qual em todo o mundo, é parte essencial dos currículos de engenharia e ciências exatas. (ÇENGEL 2014, p.1). Para o estudo e desenvolvimento deste tema faz-se necessário um bom entendimento de cálculo diferencial e integral onde deve estar claro aos estudantes vários temas, tais como variáveis dependentes e independentes, funções contínuas e descontínuas, derivadas ordinárias e parciais, diferenciais e incrementos, e integração.

De acordo com Diacu (2013), a teoria das equações diferenciais possui três grupos principais que envolvem métodos exatos, numéricos e qualitativos. Mas pode-se acrescentar a esta classificação o processo de modelagem, que trata da obtenção de uma equação diferencial que descreve certo fenômeno e interpreta os resultados de suas análises dentro de uma estrutura do modelo. Classificam-se as equações diferenciais por tipo, ordinária ou parcial, ordem ou linearidade.

Diferente das incógnitas das equações algébricas, que são números, as incógnitas das equações diferenciais são funções. Resolver uma equação diferencial significa encontrar todas as soluções, i.e., todas as funções que satisfazem a equação. (DIACU, 1959, p. 1.).

As equações diferenciais surgem quando se aplicam as leis e os princípios relevantes da física a um problema, considerando infinitas variáveis e variações de interesse.

\footnotetext{
Nas ciências e na engenharia, modelos matemáticos são desenvolvidos para auxiliar na compreensão de fenômenos físicos. Estes modelos frequentes geram uma equação que contém algumas derivadas de uma função desconhecida. Tal equação é chamada de Equação diferencial. (NAGLE, 2012, p. 1.)
}

Desta forma ao se obter uma equação diferencial que direciona uma aplicação, esta requer conhecimento adequado sobre a natureza do problema, assim como as variáveis envolvidas, as suposições aplicadas afim da simplificação, as leis e os princípios da física aplicados. Este processo para a obtenção das equações diferenciais em algumas áreas é demonstrado por meio de exemplos, ou seja, através de uma modelagem matemática. 
Para alcançar os objetivos deste artigo, o mesmo encontra-se estruturado como segue. A introdução que apresenta a justificativa, os objetivos e a problemática. Na seção 2, faz-se a modelagem matemática seguida da Lei de Resfriamento de Newton propriamente dita na seção 3. Algumas aplicações são apresentadas na seção 4; Os métodos numéricos clássicos, assim como a comparação da solução encontrada pelos mesmos e pela solução encontrada analiticamente, na seção 5 e finalmente, conclui-se o artigo na seção 6.

\title{
2. MATERIAIS E MÉTODOS
}

\subsection{Modelagens matemática}

Segundo Boyce (1930, p.38), as equações diferenciais são de interesse para não matemáticos, principalmente por causa da possibilidade de serem usadas para investigar uma variedade de problemas nas ciências físicas, biológicas e sociais. Essas equações permitem, muitas vezes, fazer previsões sobre como os processos naturais se comportarão em diversas circunstâncias. Muitas vezes é fácil permitir a variação dos parâmetros no modelo matemático em um amplo intervalo, enquanto isso poderia levar muito tempo ou ser muito caro, se não impossível, em um ambiente experimental. De qualquer modo, a modelagem matemática e a experimentação ou observação são criticamente importantes e têm papéis complementares nas investigações científicas. Modelos matemáticos são validados comparando-se suas previsões com resultados experimentais. Por outro lado, análises matemáticas podem sugerir as direções mais promissoras para exploração experimental e podem indicar, com boa precisão, que dados experimentais serão mais úteis. "Muitos fenômenos naturais podem ser modelados matematicamente. Em geral, modelar é uma tarefa difícil que requer conhecimento interdisciplinar e habilidades." (DIACU, 1959, p.4.).

Independente do campo específico de aplicação das equações diferenciais, quando nos referimos a uma modelagem matemática, de acordo com Boyce (1930, p.39) tem-se que percorrer três importantes passos:

a) Construção do modelo: nesta etapa precisa-se traduzir a situação física em expressões matemáticas.

\begin{abstract}
É importante compreender que as equações matemáticas são, quase sempre, apenas uma descrição aproximada do processo real.... Assim, você deve estar sempre atento às limitações do modelo, de modo a só usá-lo quando for razoável acreditar em sua precisão. De maneira alternativa, você poderia adotar o ponto de vista de que as equações matemáticas descrevem exatamente as operações de um modelo físico simplificado ou ideal, que foi construído (ou imaginado) de maneira a incorporar as características mais importantes do processo real. (BOYCE, 1930, p.39).
\end{abstract}

b) Análise do modelo: neste estágio, uma vez formulado matematicamente o problema, pode-se, muitas vezes, encontrar problemas nas tentativas de resolver as equações diferenciais, tornando-se neste caso necessário algumas aproximações ou adaptações, mas "é claro que tais aproximações também têm que ser examinadas sob o ponto de vista físico, para se ter clareza de que o problema matemático simplificado ainda reflete as características essenciais do problema físico que esta sendo investigado". (BOYCE, 1930, p.39).

Este processo entre a compreensão do problema e as limitações referentes ao conhecimento das técnicas matemáticas para resolvê-lo é característico da matemática aplicada, e essencial para uma construção de modelos matemáticos confiáveis, úteis e de sucesso para resoluções de problemas diversos. 
c) Comparação com Experimentos ou Observações: aqui estão relatadas as informações referentes às soluções encontradas, podendo esta ser cabível a uma verificação. Para Boyce (1930, p. 39) é claro que o fato de que a solução matemática parecer ser razoável não garante que está correta. Após a aplicação, após observar-se e em caso de o modelo matemático ser seriamente inconsistente com as observações do sistema físico que o modelo supostamente descreve é necessário verificar os erros cometidos durante a resolução do problema matemático, para assim realizar observações com mais cuidado, refinando-o.

Após estas notações referentes ao caminho que se devem seguir para a construção de um modelo matemático, os cuidados que se deve ter, segue neste artigo a resolução, através das equações diferenciais, da lei de resfriamento de Newton, na tentativa de responder o questionamento inicial, tendo em vista que esta resolução envolve uma modelagem matemática.

\subsection{Taxa de Variação de Temperatura - Lei de resfriamento de Newton}

Para resolver a problemática deste artigo, que envolve o problema de taxa de variação de temperatura, ou seja, a Lei de resfriamento de Newton, apresenta-se sua solução analítica através do uso da técnica de separação de variáveis.

Diversos autores, tais como Nagle (2012, p. 34), Çengel (2014, p. 5), Diacu (1959, p. 17), Boyce (1930, p. 7) e Zill (2012, p. 22), relatam que a lei de resfriamento de Newton descreve que a taxa segundo a qual a temperatura de um corpo varia é proporcional à diferença entre a temperatura do corpo e a temperatura do meio a qual o corpo está inserido, representada pela equação:

$$
\frac{d T}{d t}=K\left(T-T_{m}\right)
$$

De acordo com a lei empírica de Newton do esfriamento/aquecimento, a taxa segundo a qual a temperatura de um corpo varia é proporcional à diferença entre a temperatura do corpo e a temperatura do meio que o rodeia, denominada temperatura ambiente. Se $\mathrm{T}(t)$ representar a temperatura de um corpo no instante $t, T_{m}$ a temperatura do meio que o rodeia e $d T / d t$ a taxa segundo a qual a temperatura do corpo varia, a lei de Newton do esfriamento/aquecimento é convertida na sentença matemática: $\frac{d T}{d t}=K\left(T-T_{m}\right)$, onde $\mathrm{K}$ é uma constante de proporcionalidade. Em ambos os casos, esfriamento ou aquecimento, se $T_{m}$ for uma constante, é logico que $K<0$. (ZILL, 2012, p. 22).

Assim sendo, pode-se dizer que a equação (1) citada por diversos autores descreve um modelo real que relata a troca de calor de um corpo com o meio onde este se encontra, e tem-se que $\frac{d T}{d t}$ é a taxa segundo a qual a temperatura do corpo varia em relação ao tempo; $T=T(t)$ é a temperatura do corpo no instante $t ; T_{m}$ é a temperatura constante do meio ambiente; $T_{m}-T$ é a diferença entre as temperaturas; $t$ é o tempo $(t \geq 0)$, e $K$ é a constante que depende da temperatura do corpo que esta sendo analisado, sendo esta de sinal negativo se a temperatura do corpo estiver diminuindo com o passar do tempo, em relação à temperatura do meio ambiente. Ao identificar a equação $\frac{d T}{d t}=K\left(T-T_{m}\right)$, que representa a lei de resfriamento de Newton, pode-se classificar esta 
equação diferencial como equação diferencial ordinária de primeira ordem do primeiro grau linear, ou ainda como equação diferencial ordinária de primeira ordem do primeiro grau variável separável.

Uma equação diferencial linear de primeira ordem pode ser expressa por $y^{\prime}+P(x) y=R(x)$, onde $P(x)$ e $R(x)$ são funções continuas no intervalo de interesse em relação à $x$. Por sua vez, uma equação diferencial é caracterizada como equação diferencial ordinária de primeira ordem do primeiro grau variável separável se, de acordo com Simmons (2008, p.10) for possível, por manipulações algébricas elementares, reescrever a equação de modo que todas as variáveis dependentes (usualmente a variável y) estejam de um lado e todas as variáveis independentes (usualmente a variável $x$ ), do outro.

Pode-se então resolver a equação (1) que representa a lei de resfriamento de Newton por ambos os métodos, escolheu-se apresentar sua resolução através da técnica de variável separável. Assim reescrevendo a equação (1) tem-se:

$$
\frac{d T}{T-T_{m}}=K d t
$$

Integrando se ambos os lados da equação (2) em função da variável tempo, tem-se a seguinte equação:

$$
\int d T / T-T_{m}=\int K d t
$$

Assim:

$$
\ln \left(T-T_{m}\right)=K t+A
$$

Aplica-se a função exponencial em ambos os membros da equação:

$$
T-T_{m}=e^{K t+A}=e^{K t} \times e^{A} \Rightarrow T(t)-T_{m}=C e^{(K t)}
$$

Conclui-se que a solução da equação diferencial dada por (1) é:

$$
T(t)=T_{m}+C e^{(K t)}
$$

Sempre que a temperatura inicial for conhecida, $T(0)=T_{0}$, obtém-se a constante C, de modo que: $T_{0}+T_{m}+C \Rightarrow C=T_{0}-T_{m}$.

Concluindo que a solução analítica da equação diferencial dada pela equação (1) $\frac{d T}{d t}=K\left(T-T_{m}\right)$ é dada por:

$$
T(t)=T_{m}+\left(T_{0}-T_{m}\right) e^{(K t)}
$$

Tem-se, dessa forma uma aplicação real de um conteúdo do cotidiano, utilizando-se de equações diferenciais de modo que se possam apresentar respostas as indagações dos estudantes, com relação à utilização dos conceitos estudados. Além da solução analítica, pode-se apresentar, em muitas situações, soluções numéricas dos problemas reais de engenharia. Métodos numéricos, em sua maioria apresentam aproximações para as soluções. Para o caso especifico de equações diferenciais existem métodos clássicos que podem ser implementados e que retomam excelentes soluções.

O objetivo de um método numérico para uma equação diferencial é obter uma coleção de pontos $\left(t_{0}, x_{0}\right),\left(t_{1}, x_{1}\right), \ldots,\left(t_{n}, x_{n}\right)$ que rastreie uma curva que estima uma porção finita do gráfico da verdadeira solução passando pelo 
ponto $\left(t_{0}, x_{o}\right)$. Quanto mais pontos obtivermos e mais próximos eles estiverem da curva real, melhor é a aproximação. (DIACU, 1959, p.36).

Na seção 2.3.3 apresentam-se dois destes métodos numéricos, o método de Euler e o método de Runge-Kutta.

\subsection{Algumas Aplicações}

Em sala de aula, na disciplina de Equações Diferenciais Ordinárias, é comum que professores apresentem modelos matemáticos como aplicações a serem resolvidas. Na sequência apresentam-se algumas destas aplicações, em especial alguns exemplos que podem ser resolvidos através da lei de variação de temperatura de Newton.

\subsubsection{Processo de Resfriamento de uma xícara de café}

Na presente aplicação após já conhecer-se sua resolução analítica por meio da equação de resfriamento de Newton, apresenta-se na seção 3 sua resolução também através dos métodos numéricos apresentados neste artigo, para comparação dos resultados.

Na seguinte aplicação admite-se que a temperatura de uma xícara de café quente segue a lei de resfriamento de Newton, e supõe-se que sua temperatura inicial é de $93,3^{\circ} \mathrm{C}$, e que logo após o café ser coado, um minuto depois, sua temperatura é de $87,7^{\circ} \mathrm{C}$. Sendo a temperatura ambiente de $21,1^{\circ} \mathrm{C}$, questiona-se em que instante a temperatura do café irá ser de $65,6^{\circ} \mathrm{C}$.

Retirando as informações do problema temos que a temperatura inicial $T(0)$ é de $93,3^{\circ} \mathrm{C}$, a temperatura no minuto seguinte $T(1)$ é $87,7^{\circ} \mathrm{C}$, a temperatura ambiente $T(m)$ é $21,1^{\circ} \mathrm{C}$ e quer se saber o tempo $(t)$ em que a temperatura é $65,5^{\circ} \mathrm{C}$, ou seja, $T(?)=65,5^{\circ} \mathrm{C}$. Substituindo os dados referentes $T(0)$ na equação $T(t)=T_{m}+C e^{(K t)}$, encontra-se o valor de $C$.

$$
T(0)=T_{m}+C e^{(K 0)} \Rightarrow 93,3=21,1+C e^{(K 0)} \Rightarrow C=72,2
$$

Assim pode-se achar o valor de $K$, substituindo $C$ encontrado, e os dados referentes à temperatura no minuto seguinte, $T(1)$, e a temperatura do ambiente, $T(m)$, na equação, $T(t)=T_{m}+C e^{(K t)}$.

$$
T(1)=T_{m}+C e^{(K t)} \Rightarrow 87,7=21,1+72,2 e^{K} \Rightarrow 66,6=72,2 e^{K} \Rightarrow 0,92=e^{K}
$$

Aplicando-se logaritmo natural em ambos os lados da equação tem-se:

$$
\ln 0,92=\ln e^{K} \Rightarrow K=-0,08
$$

Encontrado o valor de $C$ aproximado (72,2), e o valor de $K$ aproximado $(-0,08)$, faz-se a substituição na equação, $T(t)=T_{m}+C e^{(K t)}$, a temperatura ambiente, $T(m)=21,1^{\circ} C$, e a temperatura de $65,6^{\circ} \mathrm{C}$ para assim encontrar o tempo, o instante em que o café atingiu esta temperatura.

$$
65,6=21,1+72,2 e^{(-0,08 t)} \Rightarrow 44,5=72,2 e^{(-0,08 t)} \Rightarrow 0,61=e^{(-0,08 t)}
$$

Aplicando-se logaritmo natural em ambos os lados da equação tem-se:

$$
-0,48=-0,08 t
$$


Logo:

$$
t \cong 6 \min
$$

Portanto aproximadamente 6 minutos após ser coado o café irá atingir a temperatura de $65,6^{\circ} \mathrm{C}$.

\subsubsection{Processo de Resfriamento de um Cadáver}

Peritos usualmente precisam descobrir o tempo de morte de determinado acontecimento. Faz-se uso da Lei de Resfriamento de Newton, porém segundo peritos alguns detalhes devem ser levados em consideração, tais como, se a pessoa perdeu muito sangue, ou se já passou muito tempo após o óbito. Eles utilizam em vários casos termômetros digitais para obter a temperatura ambiente e também se retira a temperatura do corpo, esta sendo medida através do reto do cadáver. Dada à temperatura ambiente, o corpo que possui a temperatura normal de $37^{\circ} \mathrm{C}$, após a morte tem sua temperatura se igualando a do ambiente. Esta temperatura normal de uma pessoa é mantida através de um equilíbrio entre o metabolismo do corpo e a temperatura ambiente. E o interessante é que estes fatos recaem em um problema que forma uma modelagem a qual será solucionada utilizandose a equação diferencial.

Uma situação problema real pode ser ocasionada através da investigação de um homicídio onde um corpo é encontrado misteriosamente às 4 horas em uma esquina entre duas ruas pouco movimentadas. $\mathrm{O}$ corpo do homem aparentava aproximadamente 30 anos. Moradores disseram que ouviram tiros por volta da meia-noite e também em torno das 3 horas da madrugada. Mas como saber a hora em que o homem foi morto? Para responder esta pergunta precisa-se saber a temperatura do corpo no instante da descoberta e a temperatura do ambiente para assim efetuar os cálculos, utilizando a equação final da temperatura em função do tempo. Para solucionar o problema admite-se que a temperatura do corpo seja $30^{\circ} \mathrm{C}$ no instante da descoberta, e que após duas horas a temperatura do corpo passa a ser de $23^{\circ} \mathrm{C}$, sabendo-se que a temperatura ambiente é de $20^{\circ} \mathrm{C}$. Primeiramente precisa-se calcular a constante $\mathrm{K}$, pois já se possui os seguintes dados:

$$
T=23^{\circ} \mathrm{C}, t=2 \text { horas }, T_{m}=20^{\circ} \mathrm{C}, T_{0}=30^{\circ} \mathrm{C} \text {. }
$$

Resolvendo através da equação $T(t)=T_{m}+\left(T_{0}-T_{m}\right) e^{(K t)}$, encontra-se o valor da constante K.

$$
\begin{gathered}
23=20+(30-20) e^{(K 2)} \\
\frac{3}{10}=e^{(K 2)} \Rightarrow \ln 0,3=\ln e^{(2 K)} \Rightarrow 2 k=-1,2 \Rightarrow K=-0,6
\end{gathered}
$$

Quer-se saber o instante da morte. Então, admitindo que a temperatura normal do corpo seja igual a $37^{\circ} \mathrm{C}$ no instante $t=0$, e a temperatura ambiente de $20^{\circ} \mathrm{C}$, calcula-se $t$ no instante da morte. Admitindo os dados na hora da morte como: $T_{0}=37^{\circ} \mathrm{C}, T_{m}=20^{\circ} \mathrm{C}, \mathrm{T}=30^{\circ} \mathrm{C}, \mathrm{K}=-0,6$, resolve-se:

$$
\begin{gathered}
T(t)=T_{m}+\left(T_{0}-T_{m}\right) e^{(K t)} \\
30=20+(37-20) e^{(-0,6 t)} \Rightarrow \frac{10}{17}=e^{-0,6 t} \Rightarrow \ln \frac{10}{17}=\ln e^{-0,6 t} \Rightarrow-0,6 t=-0,53 \Rightarrow t=0,833333 \ldots
\end{gathered}
$$


Logo té de aproximadamente $53 \mathrm{~min}$, e assim pode-se responder ao questionamento inicial, sabendo que o corpo foi encontrado às 4 horas, e já fazia 53 min que o corpo estava ali, admite-se que a hora da morte foi aproximadamente às 3 horas e sete minutos.

Esta seção apresenta recursos matemáticos sendo utilizados na prática com intuito de solucionar problemas encontrados no cotidiano.

\subsection{Métodos Numéricos para Equação Diferencial Ordinária}

Ao escolher um método a ser utilizado, procura-se aquele que é mais adequado para o problema em análise assim como as vantagens que cada método oferece juntamente com as limitações que eles apresentam. Os métodos aqui apresentados são o método de Euler e o método de Runge-Kutta.

\subsubsection{Método de Euler}

Este método é o mais velho e mais simples dos métodos numéricos e foi desenvolvido por Leonhard Euler (1707-1783) por volta de 1768. De acordo com Nagle (2012, p.19) "o método de Euler (ou o método da linha tangente) é um procedimento para construir soluções aproximadas a um problema de valor inicial para uma equação diferencial de primeira ordem”,

Considera-se o problema de valor inicial de primeira ordem,

$$
\left\{\begin{array}{c}
y^{\prime}=f(x, y) \\
y\left(x_{0}\right)=y_{0}
\end{array}\right.
$$

onde percebe-se que a função $f(x, y)$ refere-se à inclinação da reta tangente em cada ponto.

Assim, o método de Euler sugere iniciar no ponto inicial $\left(x_{0}, y_{0}\right)$ e seguir na linha reta de inclinação $f\left(x_{0}, y_{0}\right)$, linha tangente, para alguma distância determinada até o ponto $\left(x_{1}, y_{1}\right)$. Em seguida reinicia-se a inclinação para o valor $f\left(x_{1}, y_{1}\right)$ e seguindo essa linha para $\left(x_{2}, y_{2}\right)$. Então se constroem as aproximações poligonais (linha de partida) até a solução. Notando-se que a medida a qual se usa espaçamentos menores entre os pontos, empregando-se vários pontos, a convergência se dá cada vez mais próxima à solução verdadeira.

Para ser mais preciso, considere que um problema qualquer de valor inicial indicado na equação (7), tem uma solução única $f(x)$ em algum intervalo centrado em $x_{0}$. Considere que $h$ seja um número positivo fixo também chamado tamanho de passo, e considere os pontos igualmente espaçados, assim tem-se $x_{n}$ definido como $x_{n}=x_{0}+n h, n=0,1,2, \ldots$

Percebe-se que a construção de valores $y_{n}$ se aproxima dos valores da solução $f\left(x_{n}\right)$ e procede da seguinte forma: no ponto $\left(x_{0}, y_{0}\right)$, a inclinação da solução para $y^{\prime}=f(x, y), \quad y\left(x_{0}\right)=y_{0}$ é dada por $\frac{d y}{d x}=f\left(x_{0}, y_{0}\right)$. Logo, a linha tangente à curva-solução no ponto inicial $\left(x_{0}, y_{0}\right)$ é dada pela equação: $y-y_{0}=m\left(x-x_{0}\right) \Rightarrow y=y_{0}+\left(x-x_{0}\right) \frac{d x}{d y}$

$$
y=y_{0}+\left(x-x_{0}\right) f\left(x_{0}, y_{0}\right)
$$


Usando essa linha tangente para aproximar $f(x)$, tem-se para o ponto $x_{1}=x_{0}+h$, o valor $f\left(x_{1}\right) \approx y_{1}:=y_{0}+h f\left(x_{0}, y_{0}\right)$.

Em seguida, para o ponto $\left(x_{1}, y_{1}\right)$, constrói-se a nova linha com inclinação dada pelo campo de direção no ponto $\left(x_{1}, y_{1}\right)$, ou seja, com inclinação igual a $f\left(x_{1}, y_{1}\right)$. Isto é, para $x_{2}=x_{1}+h$ tem-se a aproximação $f\left(x_{2}\right) \approx y_{2}:=y_{1}+h f\left(x_{1}, y_{1}\right)$, e assim sucessivamente, repetindo-se $\mathrm{o}$ processo obtém-se os próximos pontos.

Em resumo, o método de Euler é dado pelos seguintes passos recursivos:

$$
\left\{\begin{array}{c}
x_{n+1}=x_{n}+h \\
f\left(x_{n+1}\right) \cong y_{n+1}=y_{n}+h f\left(x_{n}, y_{n}\right) \\
n=1,2,3, \ldots
\end{array}\right.
$$

O método de Euler é muito atraente por sua simplicidade, mas, em geral, apresentam aproximações menos refinadas de modo que apresenta-se na seção seguinte o método de RungeKutta.

\subsubsection{Método de Runge-Kutta}

Para se obter resultados melhores que os encontrados pelo método de Euler tem-se o método de Runge-Kutta, que alguns autores trazem também como o método de Euler refinado. Este método pode ser utilizado para ordens maiores, foi desenvolvido em 1895 pelo matemático e físico alemão Carl Runge (1856-1927) e aperfeiçoado para ordens superiores em 1901 por outro matemático alemão, Wilherm Kutta (1867-1944). Em lugar de aproximar $f(x, y)$ pelo valor na extrema esquerda do intervalo, como faz o processo de Euler, o método Runge-Kutta de segunda ordem, por exemplo, toma a média dos valores aproximados de $f(x, y)$ em ambas as extremidades do intervalo.

A beleza dos métodos de Runge-Kutta está no fato de que eles oferecem a exatidão melhor que de outros métodos, porém sem a necessidade de derivadas. A versão que se mostra em seguida através das fórmulas recursivas é também chamada de método do ponto intermediário.

$$
\left\{\begin{array}{l}
x_{n+1}=x_{n}+h \\
y_{n+1}=y_{n}+h f\left(x_{n}+\frac{h}{2}, y_{n}+\frac{h}{2} f\left(x_{n}, y_{n}\right)\right)
\end{array}\right.
$$

Porém os mais populares métodos de Runge-Kutta são os de quarta ordem, disponível em várias versões. De modo geral, ele produz aproximações muito precisas mesmo quando o número de iterações é razoavelmente pequeno. Como no método de Euler, o número de passos esta relacionado ao tamanho do passo $h$.

A fórmula de Runge-Kutta parece ser mais complicada que a fórmula de Euler, entretanto, por causa de exatidão e da facilidade de uso, o método de Runge-Kutta é o preferido para ser aplicado. Para a resolução do problema dado pelas equações (7) faz-se uso do método de quarta ordem de Runge-Kutta que consiste nas seguintes fórmulas recursivas:

$$
\left\{\begin{array}{l}
x_{n+1}=x_{n}+h \\
y_{n+1}=y_{n}+\frac{1}{6}\left(k_{1}+2 k_{2}+2 k_{3}+k_{4}\right)
\end{array}\right.
$$


onde:

$$
\left\{\begin{array}{l}
k_{1}=h f\left(x_{n}, y_{n}\right), \\
k_{2}=h f\left(x_{n}+\frac{h}{2}, y_{n}+\frac{k_{1}}{2}\right), \\
k_{3}=h f\left(x_{n}+\frac{h}{2}, y_{n}+\frac{k_{2}}{2}\right), \\
k_{4}=h f\left(x_{n}+h, y_{n}+k_{3}\right) .
\end{array}\right.
$$

Ao realizar estes cálculos aconselha-se observar e tomar cuidado, pois se percebe que $k_{2}$ depende de $k_{1}, k_{3}$ depende de $k_{2}$ e $k_{4}$ depende de $k_{3}$.

\section{COMPARANDO OS MÉTODOS NUMÉRICOS PRESENTES NESTE ARTIGO}

Agora faz-se uso dos métodos numéricos apresentados nas seções 2.3.3.1 e 2.3.3.2, métodos de Euler e de Runge-Kutta, para resolver a equação da situação problema dada na seção 2.3.1 resolvida através da equação de Resfriamento de Newton, e assim comparar e retirar as conclusões possíveis nas três resoluções.

Para ambos os métodos numéricos de resolução admite-se o valor da constante $K$ igual á $-0,08$, valor encontrado anteriormente, e $h$, o valor do passo a ser tomado igual a 0,5. Em um intervalo de tempo de $[0,10]$ minutos em uma temperatura ambiente de $21,1^{\circ} \mathrm{C}$. Assim sendo, para o método de Euler utilizam-se das equações (9), dadas por $f(t, T)=K\left(T_{0}-T_{m}\right)$, e $T_{n}=T_{0}+h f\left(t_{0}, T_{0}\right)$, e através dos cálculos referentes encontra-se a temperatura no respectivo tempo determinado.

Como já se sabe em $t=0$ tem-se $T_{0}=93,3$ (temperatura inicial igual a $93,3^{\circ} \mathrm{C}$ ), podendo assim encontrar através da equação $T_{n}=T_{0}+h f\left(t_{0}, T_{0}\right)$, a temperatura relativa a tempo seguinte, ou seja, $T(1)$ com o tempo $t=0,5$.

$$
T_{1}=T_{0}+h f\left(t_{0}, T_{0}\right)
$$

Como:

$$
\begin{gathered}
f\left(t_{0}, T_{0}\right)=K\left(T_{0}-T_{m}\right) \\
f\left(t_{0}, T_{0}\right)=-0,08(93,3-21,1) \\
f\left(t_{0}, T_{0}\right)=-5,776
\end{gathered}
$$

Substituindo este valor encontrado em $T_{1}=T_{0}+h f\left(t_{0}, T_{0}\right)$, tem-se:

$$
\begin{gathered}
T_{1}=93,3+0,5 \cdot(-5,776) \\
T_{1}=90,412
\end{gathered}
$$

Encontra-se assim a temperatura de $90,412^{\circ} C(T(1)=90,412)$, referente ao tempo $t_{1}=0,5$. O método numérico de Euler irá ser utilizado para encontrar a temperatura seguinte, $T(2)$, com o tempo $t_{2}=1 \mathrm{~min}$. Aplicando-se o mesmo procedimento anterior. 
Substituindo o valor encontrado em $T_{2}=T_{1}+h f\left(t_{1}, T_{1}\right)$ encontra-se:

$$
\begin{gathered}
T_{2}=90,412+0,5 \cdot(-5,54496) \\
T_{2}=87,63952
\end{gathered}
$$

Realizando os cálculos referentes ao intervalo de tempo de 0,10 minutos, determinados anteriormente com $h=0,5$, ou seja, o tamanho do passo igual a 0,5 pode-se construir a Tabela (1) com as temperaturas encontradas nos respectivos tempos, através da utilização do método de Euler.

\begin{tabular}{|c|c|c|}
\hline Tempo $(t)(\min )$ & $f(t, T)=K\left(T_{0}-T_{m}\right)$ & $T_{n}=T_{0}+h f\left(t_{0}, T_{0}\right) \quad\left({ }^{\circ} C\right)$ \\
\hline$t_{0}=0$ & $\longrightarrow$ & 93,3 \\
\hline$t_{1}=0,5$ & $-5,776$ & 90,412 \\
\hline$t_{2}=1$ & $-5,54496$ & 87,63952 \\
\hline$t_{3}=1,5$ & $-5,3231616$ & 84,9779392 \\
\hline$t_{4}=2$ & $-5,110235136$ & 82,42282163 \\
\hline$t_{5}=2,5$ & $-4,905825731$ & 79,96990876 \\
\hline$t_{6}=3$ & $-4,709592701$ & 77,61511241 \\
\hline$t_{7}=3,5$ & $-4,521208993$ & 75,35450791 \\
\hline$t_{8}=4$ & $-4,340360633$ & 73,18432759 \\
\hline$t_{9}=4,5$ & $-4,166746207$ & 71,10095449 \\
\hline$t_{10}=5$ & $-4,000076359$ & 69,10091631 \\
\hline$t_{11}=5,5$ & $-3,8400773305$ & 67,18087966 \\
\hline$t_{12}=6$ & $-3,686470373$ & 65,33764447 \\
\hline$t_{13}=6,5$ & $-3,539011558$ & 63,56813869 \\
\hline$t_{14}=7$ & $-3,397451095$ & 61,86941314 \\
\hline$t_{15}=7,5$ & $-3,261553051$ & 60,23863661 \\
\hline$t_{16}=8$ & $-3,131090929$ & 58,67309115 \\
\hline$t_{17}=8,5$ & $-3,005847292$ & 57,1701675 \\
\hline$t_{18}=9$ & $-2,8856134$ & 55,7273608 \\
\hline$t_{19}=9,5$ & $-2,770188864$ & 54,34226637 \\
\hline$t_{20}=10$ & $-2,659381309$ & 53,01257572 \\
\hline
\end{tabular}

Tabela 1- Método Numérico de Euler

Fonte: Elaborado pelas autoras (2015).

Já para os cálculos do método de Runge-Kutta de quarta ordem, que se apresenta a seguir, além da equação $f(t, T)=K\left(T_{0}-T_{m}\right)$, e dos dados anteriormente encontrados, faz-se uso da 
seguinte equação, $\quad T_{n+1}=T_{n}+\frac{h}{6}\left(k_{1}+2 k_{2}+2 k_{3}+k_{4}\right), \quad$ lembrado que $\quad k_{1}=f\left(t_{n}, T_{n}\right)$, $k_{2}=f\left(t_{n}+\frac{h}{2}, T_{n}+\frac{k_{1}}{2}\right), k_{3}=f\left(t_{n}+\frac{h}{2}, T_{n}+\frac{k_{2}}{2}\right), k_{4}=f\left(t_{n}+h, T_{n}+k_{3}\right)$.

Com a temperatura inicial de $93,3^{\circ} \mathrm{C}\left(T_{0}=93,3\right)$ encontra-se $T_{1}$, a temperatura relativa ao primeiro tempo determinado pelo tamanho do passo, $h=0,5$, ou seja, $t_{1}=0,5$, utilizando-se a equação $T_{n+1}=T_{n}+\frac{h}{6}\left(k_{1}+2 k_{2}+2 k_{3}+k_{4}\right)$, calcula-se $T_{1}$, mas, precisam-se encontrar primeiramente os valores respectivos de $k_{1}, k_{2}, k_{3}$ e $k_{4}$. De acordo com o método de Runge-Kutta:

$$
\begin{gathered}
k_{1}=f\left(t_{0}, T_{0}\right) \Rightarrow k_{1}=-5,776 \\
k_{2}=f\left(t_{n}+\frac{h}{2}, T_{n}+\frac{k_{1}}{2}\right) \Rightarrow k_{2}=f\left(t_{0}+\frac{0,5}{2}, T_{0}+\frac{-2,888}{2}\right) \Rightarrow k_{2}=f(0+0,25 ; 93,3-1,444) \Rightarrow \\
\Rightarrow k_{2}=f(0,25 ; 91,856)
\end{gathered}
$$

Calculando $f(0,25 ; 91,856)$ tem-se:

$$
f(t, T)=K\left(T-T_{m}\right) \Rightarrow f(0,25 ; 91,856)=-0,08(91,856-21,1) \Rightarrow f(0,25 ; 91,856)=-5,66048
$$

Logo,

$$
k_{2}=-5,66048
$$

Calculando-se $k_{3}$ :

Calculando $f(0,25 ; 91,88488)$ tem-se:

$f(t, T)=K\left(T-T_{m}\right) \Rightarrow f(0,25 ; 90,88488)=-0,08(90,88488-21,1) \Rightarrow f(0,25 ; 87,63952)=-5,6627904$

Logo,

$$
k_{3}=-5,6627904
$$

Encontrando $k_{4}$ :

$k_{4}=f\left(t_{0}+h, T_{0}+k_{3}\right) \Rightarrow k_{4}=f(0+0,5 ; 93,3+(-2,8313952)) \Rightarrow k_{4}=f(0,5,90,4686048)$.

Calculando $f(0,5,90,4686048)$ tem-se:

$f(t, T)=K\left(T-T_{m}\right) \Rightarrow f(0,25 ; 90,4686048)=-0,08(90,4686048-21,1) \Rightarrow f(0,25 ; 90,4686048)=-5,549488384$.

Logo, $\quad k_{4}=-5,549488384$. Assim pode-se voltar à equação $T_{1}=T_{0}+\frac{h}{6}\left(k_{1}+2 k_{2}+2 k_{3}+k_{4}\right)$, e substituir os valores encontrados para obter o valor de $T_{1}$.

$T_{1}=93,3+\frac{0,5}{6}(-5,776+2(-5,66048)+2(-5,6627904)+(-5,549488384))$

$T_{1}=90,46899757$ 
Depois de encontrado $T_{1}$, o processo é repetido sucessivamente para assim encontrar $T_{2}$, sempre calculando-se os valores de $k_{1}, k_{2}, k_{3}$ e $k_{4}$, repetindo-se o processo anterior, ou seja, $T_{2}=T_{1}+\frac{h}{6}\left(K_{1}+2 K_{2}+2 K_{3}+K_{4}\right)$, onde:

$$
\begin{aligned}
& k_{1}=f\left(t_{1}, T_{1}\right), \\
& k_{2}=f\left(t_{1}+\frac{h}{2}, T_{1}+\frac{k_{1}}{2}\right), \\
& k_{3}=f\left(t_{1}+\frac{h}{2}, T_{1}+\frac{k_{2}}{2}\right), \\
& k_{4}=f\left(t_{1}+h, T_{1}+k_{3}\right) .
\end{aligned}
$$

Como se segue na tabela (2) (Método Numérico de Runge-Kutta), todos os cálculos realizados em cada tempo respectivamente dado, referente ao passo, $h=0,5$, dado:

Tabela 2- Método Numérico de Runge-Kutta

\begin{tabular}{c|c|c|c|c|c}
\hline $\begin{array}{c}\text { Tempo } \\
\left(t_{n}\right) \\
(\mathrm{min})\end{array}$ & $k_{1}$ & $k_{2}$ & $k_{3}$ & $k_{4}$ & $T_{n+1}=T_{n}+\frac{h}{6}\left(k_{1}+2 k_{2}+2 k_{3}+k_{4}\right)$ \\
$\left({ }^{\circ} \mathrm{C}\right)$
\end{tabular}




\begin{tabular}{c|c|c|c|c|c}
$t_{12}=6$ & $-3,719954404$ & $-3,502611050$ & $-3,64704329$ & $-3,574072672$ & 65,77616136 \\
\hline$t_{13}=6,5$ & $-3,570929089$ & $-3,365271709$ & $-3,50404068$ & $-3,433931281$ & 64,02438405 \\
\hline$t_{14}=7$ & $-3,433950724$ & $-3,233175216$ & $-3,36664529$ & $-3,299284912$ & 62,34129491 \\
\hline$t_{15}=7,5$ & $-3,299303593$ & $-3,106537330$ & $-3,23463724$ & $-3,169918103$ & 60,72420065 \\
\hline$t_{16}=8$ & $-3,169936052$ & $-2,984728262$ & $-3,10780530$ & $-3,045623839$ & 59,17051355 \\
\hline$t_{17}=8,5$ & $-3,045641084$ & $-2,867695395$ & $-2,98594651$ & $-2,926203223$ & 57,67774739 \\
\hline$t_{18}=9$ & $-2,926219791$ & $-2,755251453$ & $-2,86886588$ & $-2,811465156$ & 56,24351338 \\
\hline$t_{19}=9,5$ & $-2,811481075$ & $-2,647216501$ & $-2,75637604$ & $-2,701226033$ & 54,86551659 \\
\hline$t_{20}=10$ & $-2,701241327$ & $-2,543417659$ & $-2,64829699$ & $-2,595309447$ & 53,54155178
\end{tabular}

Fonte: Elaborado pelas autoras (2015).

Os cálculos foram realizados com auxílio do software visual cálculo numérico utilizando-se nove casas decimais. Pode-se então realizar uma comparação entre o valor analítico (exato) da temperatura, estabelecido pela lei de resfriamento de Newton, e os métodos numéricos de Euler e de Runge-Kutta.

Tabela 3- Comparação entre métodos analisados

\begin{tabular}{c|c|c|c}
\hline $\begin{array}{c}\text { Tempo }(t) \\
(\min )\end{array}$ & $\begin{array}{c}\text { Valor Analítico (exato) } \\
T(t)=T_{m}+C e^{(K t)}\left({ }^{\circ} \mathrm{C}\right)\end{array}$ & $\begin{array}{c}\text { Método de Euler } \\
\left.T_{n}=T_{0}+h f\left(t_{0}, T_{0}\right){ }^{\circ} \mathrm{C}\right)\end{array}$ & $\begin{array}{c}\text { Método de Runge-Kutta } \\
T_{n+1}=T_{n}+\frac{h}{6}\left(k_{1}+2 k_{2}+2 k_{3}+k_{4}\right){ }^{\circ} \mathrm{C}\end{array}$ \\
\hline$t_{1}=0,5$ & 90,46899751 & 90,412 & 90,46899756 \\
\hline$t_{2}=1$ & 87,74900021 & 87,63952 & 87,74900032 \\
\hline$t_{3}=1,5$ & 85,13565553 & 84,9779392 & 85,13565570 \\
\hline$t_{4}=2$ & 82,62478156 & 82,42282163 & 82,62478178 \\
\hline$t_{5}=2,5$ & 80,21236037 & 79,96990876 & 80,21236063 \\
\hline$t_{6}=3$ & 77,89453157 & 77,61511241 & 77,89453186 \\
\hline$t_{7}=3,5$ & 75,66758613 & 75,35450791 & 73,66758647 \\
\hline$t_{8}=4$ & 73,52796048 & 73,18432759 & 71,47223114 \\
\hline$t_{9}=4,5$ & 71,47223074 & 71,10095449 & 69,49710775 \\
\hline$t_{10}=5$ & 69,49710732 & 69,10091631 & 67,59943005 \\
\hline$t_{11}=5,5$ & 67,5994296 & 67,18087966 & $\mathbf{6 5 , 7 7 6 1 6 1 3 6}$ \\
\hline$t_{12}=6$ & $\mathbf{6 5 , 7 7 6 1 6 0 8 9}$ & $\mathbf{6 5 , 3 3 7 6 4 4 4 7}$ & \\
\hline
\end{tabular}

Fonte: Elaborado pelas autoras (2015).

2:

Traduzindo e reescrevendo os dados acima na forma da representação gráfica temos a figura 


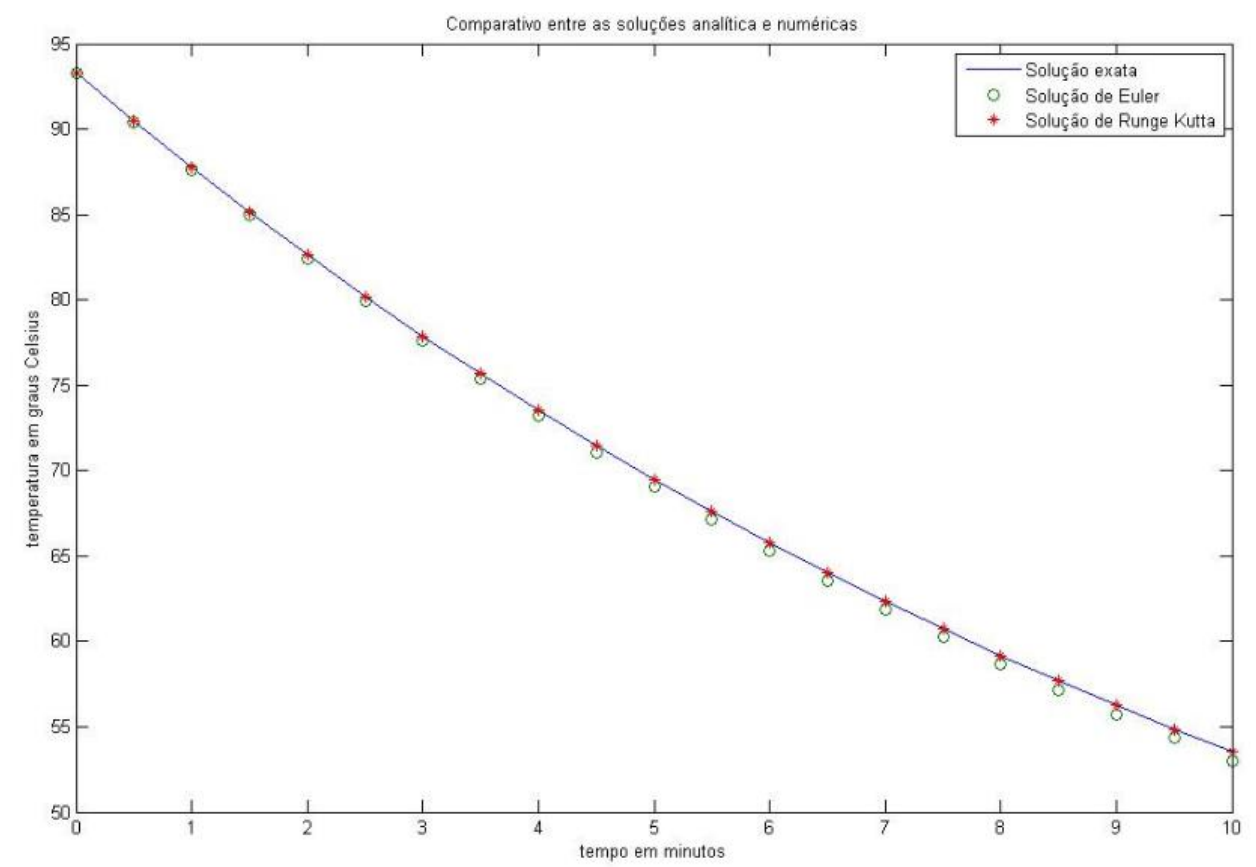

Figura 2- Gráfico de comparação dos métodos apresentados neste artigo

Fonte: Elaborado pelas autoras (2015).

Pode-se concluir através dos cálculos e do gráfico, a eficiência do método de Runge-Kutta, o qual se aproxima muito do valor exato da equação referente ao problema da aplicação inicial, resolvido analiticamente.

\section{CONCLUSÃO}

O presente artigo buscou salientar a matemática aliada aos conhecimentos práticos do cotidiano satisfazendo e respondendo as inquietações e interrogações muitas vezes realizadas pelos estudantes. Espera-se assim que este venha contribuir mostrando que sim, é possível trabalhar com os estudantes conteúdos que muitas vezes quando ensinados podem parecer de difícil compreensão, e até mesmo "vago", através de aplicações que envolvem o conhecimento vivido diariamente por eles mesmos, se tornando assim a aprendizagem satisfatória e com o verdadeiro sentido na qual os estudantes muitas vezes buscam nas salas de aula.

Por fim, neste artigo, fez a resolução da equação diferencial de primeira ordem do primeiro grau escolhida (Lei de Resfriamento de Newton) utilizando primeiramente a resolução analítica através da técnica de separação de variáveis e por seguinte sua resolução pelos métodos numéricos de Euller e Runge-Kutta, comparando-se assim seus resultados. De modo que, uma forma de resolução completa a outra, contemplando uma aprendizagem que faz sentido, com aplicações que trazem mais significados à aprendizagem de modo a não representar uma aprendizagem solta, com cálculos sem preocupação com a realidade. Os resultados obtidos com os métodos numéricos e a resolução analítica se mostraram eficazes na solução do problema. 


\section{REFERENCIAS}

BOYCE, William E; Richard C. DiPrima.Equações Diferenciais Elementares e Problemas de Valores de Contorno. Rio de Janeiro. LTC. 2014.

ÇENGEL, Yunus A., William J. Palm III. Equações Diferenciais. Porto Alegre. AMGH Editora Ltda. 2014.

DIACU, Florin. Introdução a Equações Diferenciais: teoria e aplicações. $2^{\text {a }}$ ed. Rio de Janeiro.LCT.1959.

NAGLE, R. Kent, Edward B. Saff, Arthur David Snider. Equações Diferenciais. São Paulo. $8^{a}$ edição. Pearson Education do Brasil, 2012.

SIMMONS, George F.; Steven G. Krantz. Equações Diferenciais: Teoria, Técnica e Prática. São Paulo. McGraw-Hill. 2008.

ZILL, Dennis G.. Equações Diferenciais com aplicação em modelagem - tradução da $9^{a}$ edição norteamericana. 2 ed. São Paulo. Cengage Learning, 2012. 